\title{
Lactobacillus demonstrate thiol-independent metabolism of methylglyoxal: Implications toward browning prevention in Parmesan cheese
}

\author{
N. N. Gandhi, ${ }^{*}$ P. F. Cobra,† J. L. Steele, ${ }^{*}$ J. L. Markley, † and S. A. Rankin*1 \\ *Department of Food Science, and \\ †Department of Biochemistry, National Magnetic Resonance Facility, University of Wisconsin, Madison 53706
}

\begin{abstract}
Endogenous production of $\alpha$-dicarbonyls by lactic acid bacteria can influence the quality and consistency of fermented foods and beverages. Methylglyoxal (MG) in Parmesan cheese can contribute toward undesired browning during low temperature ripening and storage conditions, leading to the economic depreciation of affected cheeses. We demonstrate the effects of exogenously added MG on browning and volatile formation using a Parmesan cheese extract (PCE). To determine the influence of Lactobacillus on $\alpha$-dicarbonyls, strains were screened for their ability to modulate concentrations of MG, glyoxal, and diacetyl in PCE. It was found that a major metabolic pathway of MG in Lactobacillus is a thiol-independent reduction, whereby MG is partially or fully reduced to acetol and 1,2-propanediol, respectively. The majority of lactobacilli grown in PCE accumulated the intermediate acetol, whereas Lactobacillus brevis 367 formed exclusively 1,2-propanediol and Lactobacillus fermentum 14931 formed both metabolites. In addition, we determined the inherent tolerance to bacteriostatic concentrations of MG among lactobacilli grown in rich media. It was found that $L$. brevis 367 reduces MG exclusively to 1,2-propanediol, which correlates to both its ability to significantly decrease MG concentrations in PCE, as well as its significantly higher tolerance to $\mathrm{MG}$, in comparison to other lactobacilli screened. These findings have broader implications toward lactobacilli as a viable solution for reducing MG-mediated browning of Parmesan cheese.
\end{abstract}

Key words: Lactobacillus, $\alpha$-dicarbonyl, methylglyoxal, Parmesan cheese browning

\section{INTRODUCTION}

The complexity of browning reactions arises partly from a series of parallel and sequential reactions stem-

Received July 26, 2017.

Accepted October 16, 2017.

${ }^{1}$ Corresponding author: sarankin@wisc.edu ming from the glycation of peptides in the Maillard reaction. Oxoaldehydes are reactive intermediates of the Maillard reaction that augment and redirect glycation, adding to this complexity. The carbonyl carbon of $\alpha$-oxoaldehydes such as glyoxal and methylglyoxal (MG) are highly electrophilic due to their oxidative state and vicinal position to a second carbonyl group. This instability enhances elimination, condensation, and other degradation mechanisms that can form stable end-stage adducts called advanced glycation end products (AGE) as well as brown, nitrogenous, polymeric substances responsible for browning known as melanoidins (Nemet et al., 2006, Ramasamy et al., 2006). Because of the reactivity of these $\alpha$-oxoaldehydes, controlling the formation of AGE and melanoidins during processing, cooking, and prolonged storage of food and beverages has remained a difficult undertaking.

The development of brown pigmentation and concomitant alteration in flavor profiles during the aging process of cheese is an enduring issue, causing economic detriment to the industry (Yann et al., 2005). Brown pigmentation may materialize during the latter ripening and storage periods of Parmesan cheese and is atypical of Maillard reactions because it occurs in the relative absence of reducing sugars and at low temperature (Divine et al., 2012). The microbial production of MG has been implicated as a major contributor toward browning defects during low-temperature storage, leading to the economic depreciation of affected cheeses (Divine and Rankin, 2013). The major source of MG in biological systems is from the degradation of the glycolytic intermediate, dihydroxyacetonephosphate (DHAP), but less is known about MG metabolism in lactic acid bacteria (LAB) with high effect on fermented food systems.

The browning defect is more prominently observed in cheeses that have undergone direct salting of the curd before pressing compared with the more time-intensive brine salting of already pressed cheese (McDonald, 1992). A directly salted cheese has a uniform dispersion of salt throughout the wheel, whereas a brined cheese has a gradient concentration of salt, with less diffusion 
Table 1. Lactobacillus strains included in this study

\begin{tabular}{lll}
\hline Lactobacillus strain & Species description & Isolation source \\
\hline L. brevis ATCC 367 & Obligate heterofermentative & Silage \\
L. casei 12 A & Facultative heterofermentative & Silage \\
L. casei ATCC 334 & Facultative heterofermentative & Dairy \\
L. curvatus $112309-12$ & Facultative heterofermentative & Dairy \\
L. fermentum ATCC 14931 & Obligate heterofermentative & Fermented beets \\
L. fermentum ATCC 8289 & Obligate heterofermentative & Unknown \\
L. helveticus CNRZ32 & Obligate heterofermentative & Dairy \\
L. plantarum Ssp. plantarum ATCC 14971 & Facultative heterofermentative & Pickled cabbage \\
L. plantarum WCFS1 & Facultative heterofermentative & Human saliva \\
L. reuteri PTA-6475 & Obligate heterofermentative & Human breast milk \\
L. reuteri SD2112 ATCC 55730 & Obligate heterofermentative & Human breast milk \\
L. rhamnosus GG ATCC 53103 & Facultative heterofermentative & Human intestine \\
L. sakei $23 \mathrm{SK}$ & Facultative heterofermentative & Meat, fish \\
L. sakei ATCC 15521 & Facultative heterofermentative & Moto, sake starter \\
\hline
\end{tabular}

toward the center. Therefore, it is likely that MG formation is linked to the discrepancy in ripening microbiota associated with the 2 manufacturing styles. Currently, natural solutions to prevent or decrease browning do not exist outside of using chemical reducing agents and bleaching applications (Divine and Rankin, 2013). As a result, there is great interest within the industry to develop a microbial solution for reducing MG-mediated browning that is amendable to the standard of identity of Parmesan cheese.

In many long-ripened cheeses, nonstarter lactic acid bacteria (NSLAB) previously existing in low levels in the milk or processing environment begin to thrive during later stages of ripening. Nonstarter lactic acid bacteria, predominated by facultative and obligate heterofermentative lactobacilli, have a significant role in cheese flavor development. Their effect on cheese flavor development can be either positive or negative; therefore, industry expends significant effort to control the NSLAB microbiota of cheese. One approach used by industry to control the NSLAB microbiota is to select NSLAB with desired properties and to add these cultures (referred to as culture adjuncts) to cheese at relatively high numbers (e.g., $1 \times 10^{5} \mathrm{cfu} / \mathrm{mL}$; Broadbent et al., 2011). If a NSLAB capable of reducing the level of MG in cheese could be identified, it may have potential as a culture adjunct for the control of MGmediated browning in cheese.

The present study demonstrates the effects of exogenously added MG on the color and volatile profile of Parmesan cheese extract (PCE). We assess the ability of different strains of Lactobacillus to modulate glyoxal, MG, and diacetyl after prolonged single-strain fermentation in PCE. Furthermore, we identify a major metabolic pathway of MG in Lactobacillus growing in $\mathrm{PCE}$ and examine strain-specific tolerance to MG.

\section{MATERIALS AND METHODS}

\section{Bacterial Strains and Growth Conditions}

Lactobacillus selected for this study are listed in Table 1. Stock cultures were maintained at $-80^{\circ} \mathrm{C}$ in de Man, Rogosa, and Sharpe (MRS) medium (BD Biosciences, Sparks, MD) containing 15\% (vol/vol) glycerol. All media containing MG was made daily. Working cultures in PCE were prepared from frozen stocks by a single transfer in MRS followed by 2 successive transfers in PCE. When screening strains for MG tolerance, working cultures of Lactobacillus were prepared from frozen stocks by 2 successive transfers in MRS. All sub-cultures and working cultures of Lactobacillus were propagated at $37^{\circ} \mathrm{C}$, without shaking, for 16 to $18 \mathrm{~h}$.

\section{Parmesan Cheese Extract}

Parmesan cheese extract was obtained from a 10-moold Parmesan cheese and processed by the Western Dairy Center at Utah State University. A Parmesan cheese block was cut into $5.08-\mathrm{cm}$ cubes and processed through a Commitrol grinder (Urschel, Chesterton, IN) using a $0.317-\mathrm{cm}$ cutting head. The ground cheese was vacuum sealed and cooled at $4^{\circ} \mathrm{C}$ for $24 \mathrm{~h}$. A minimum of $54.4 \mathrm{~kg}$ of cheese was combined with $265 \mathrm{~L}$ of deionized water in a stainless-steel, steam-heated, jacketed vat with a total capacity of $321 \mathrm{~L}$. The cheese and water mixture was continuously stirred and heated from 15.5 to $48.8^{\circ} \mathrm{C}$ over $40 \mathrm{~min}$ and held at $48.8^{\circ} \mathrm{C}$ for $20 \mathrm{~min}$. Cheese solids were removed from the cheese-water extract by processing through a cheesecloth. The extract $(\sim 265 \mathrm{~L})$ was processed through 2 spiral-wound UF membranes with a MW cutoff of 5,000 kDa while using an operating flow of $18.9 \mathrm{~L} / \mathrm{min}$, operating temperature 
of $48.8^{\circ} \mathrm{C}$, and operating pressure of 100 to $1,000 \mathrm{kPa}$. The permeate was collected and recycled once with the concentrated retentate through the UF system before a final reverse osmosis filtration treatment. The reverse osmosis filtration system used the aforementioned conditions albeit at an operating pressure of 3,000 to 6,000 $\mathrm{kPa}$. The extract was packaged and stored at $-20^{\circ} \mathrm{C}$. Prior to use as a bacterial growth media, PCE was thawed, filter sterilized $(0.2-\mu \mathrm{m}$ filter, Thermo Scientific Bottle-top Nalgene), and stored at $4^{\circ} \mathrm{C}$.

\section{Identification of Volatiles Formed from Exogenous Addition of MG to PCE by Solid-Phase Microextraction GC-MS}

Increasing concentrations of MG were added to PCE and incubated at $65^{\circ} \mathrm{C}$ for $24 \mathrm{~h}$ to accelerate reactivity. Samples were visualized in cuvettes placed against a light source. A browning index (defined as the optical density difference measured at 420 and $500 \mathrm{~nm}$ ) was used to define positive discoloration. In parallel, volatile profiles of PCE containing either $0,1,10,50$, or $100 \mathrm{mM}$ MG were determined using solid-phase microextraction (SPME) and GC-MS. Ten-milliliter samples were prepared in $16 \times 60 \mathrm{~mm}$ screw-cap glass vials with rubber septums (Fisher Scientific, Pittsburgh, PA) and heated at $65^{\circ} \mathrm{C}$ for $24 \mathrm{~h}$ to accelerate reactivity. Volatiles were trapped by exposing an $85-\mu \mathrm{m}$ polydimethylsiloxane/ Carboxen SPME fiber (Supelco, Bellefonte, PA) to the head space for $20 \mathrm{~min}$ at $40^{\circ} \mathrm{C}$. The initial temperature of the front inlet was $220^{\circ} \mathrm{C}$ at a flow of $33.9 \mathrm{~mL} /$ min. The sample was purged at $30.0 \mathrm{~mL} / \mathrm{min}$ with a preliminary holding time of $5 \mathrm{~min}$, using helium as the carrier gas. The oven temperature was held at $40^{\circ} \mathrm{C}$ for $4 \mathrm{~min}$ and increased by $10^{\circ} \mathrm{C} / \mathrm{min}$ to $180^{\circ} \mathrm{C}$ for a final isothermal period of $3 \mathrm{~min}$. Chemstation Software (Rev. D.02.00 SP1, Agilent Technologies Inc.) was used to identify volatiles by matching the mass spectra with a published mass spectral database (National Institute of Standards and Technology, version 1.7, Agilent Technologies Inc.). Samples were prepared in duplicate and compounds with greater than $70 \%$ spectral matches were reported if an increase in abundance was detected in correlation with increased MG concentration.

\section{Quantification of Extracellular a-Dicarbonyls in PCE by GC-MS}

Analyte concentrations of glyoxal, MG, diacetyl, and 2,3 hexanedione (internal standard) were measured by derivatization with 1,2-diaminobenzene and GC-MS using methods adapted from Chaplen et al. (1996) and the Compendium of International Analysis of Methods (2010). Early stationary-phase cells were inoculated $(0.1 \% \mathrm{vol} / \mathrm{vol})$ into $50 \mathrm{~mL}$ of PCE containing $1 \mathrm{mM}$ MG and incubated at $37^{\circ} \mathrm{C}$ for $1 \mathrm{wk}$. Added to each $50-$ $\mathrm{mL}$ sample was $17.5 \mathrm{~m} M 2$,3-hexanedione. Each sample was treated with $1.94 \mathrm{~mL}$ of $70 \%$ perchloric acid and incubated on ice for $30 \mathrm{~min}$ before being centrifuged at $11,400 \times g$ for $10 \mathrm{~min}$ at $4^{\circ} \mathrm{C}$. Supernatants were separated from cell pellets and allowed to reach room temperature before being brought to a $\mathrm{pH}$ of 8.0 using $\mathrm{NaOH}$. Added to each sample was $20 \mathrm{mg}$ of 1,2 -diaminobenzene. Derivatization occurred at $60^{\circ} \mathrm{C}$ for 3 $\mathrm{h}$ before samples were brought to room temperature and a $\mathrm{pH}$ of 2.0 using $\mathrm{H}_{2} \mathrm{SO}_{4}$. Liquid-liquid extraction was performed twice using $5 \mathrm{~mL}$ of dichloromethane and decanting the lower phase using a separatory funnel. Extracts were dried over $1 \mathrm{~g}$ of anhydrous $\mathrm{Na}_{2} \mathrm{SO}_{4}$, decanted, and syringe filtered into 2-mL GC-MS vials. Commercial solutions of $\mathrm{MG}\left(\sim 40 \%\right.$ in $\left.\mathrm{H}_{2} \mathrm{O}\right)$, glyoxal, diacetyl, 2,3-hexanedione, methylquinoxaline, 2-methylquinoxaline, 2,3-methylquinoxaline, 2,3-diethylquinoxaline, and tablets of 1,2-diaminobenzene were purchased from Sigma-Aldrich (St. Louis, MO).

The gas chromatograph (Agilent 6890N, Agilent Technologies Inc., Palo Alto, CA) was fitted with a fused-silica capillary column (RTx-5MS, $30 \mathrm{~m}$ long $\times$ $0.25 \mathrm{~mm}$ i.d. $\times 0.5 \mu \mathrm{m}$ film thickness; Restek Corp., Bellefonte, PA) and paired with a mass selective detector (Agilent $5973 \mathrm{MS}$, Agilent Technologies Inc.). The initial temperature of the front inlet was $250^{\circ} \mathrm{C}$ at a flow of $40.0 \mathrm{~mL} / \mathrm{min}$, using helium as the carrier gas. The volume injected was $1 \mu \mathrm{L}$, splitless. The oven temperature was held at $60^{\circ} \mathrm{C}$ for $1 \mathrm{~min}$, then programmed to increase at a rate of $10^{\circ} \mathrm{C} / \mathrm{min}$ to $220^{\circ} \mathrm{C}$ with a final isothermal period of $10 \mathrm{~min}$. Chemstation Software (Rev. D.02.00 SP1, Agilent Technologies Inc.) was used to identify quinoxaline, 2-methylquinoxaline, 2,3-methylquinoxaline, and 2,3-diethylquinoxaline representing glyoxal, MG, diacetyl, and 2,3-hexanedione, respectively. Analytes were confirmed by comparison of retention times to purchased standards and the selected ion monitoring method; glyoxal: ion mass to charge ratio $(\boldsymbol{m} / \boldsymbol{z})=103$ and 130 , MG: ion $m / z=117$ and 144, diacetyl: ion $m / z=117$ and 158, 2,3-hexanedione: ion $m / z=158$ and 171 . The quantitative determination of dicarbonyl compounds was performed using a multiple-point external standard curve of quinoxalines from $10 \mu M$ to $2 \mathrm{~m} M$ and analyte concentrations were normalized to the maximum optical density at $600 \mathrm{~nm}$ $\left(\mathbf{O D}_{600}\right)$ achieved by each strain. Analyses were performed in biological triplicate, and strains unable to propagate in PCE were omitted from this analysis. 


\section{Identification and Quantification of MG Metabolites in PCE by ${ }^{1} \mathrm{H}$-Nuclear Magnetic Resonance}

Cultures of Lactobacillus in PCE containing $6 \mathrm{mM}$ MG were inoculated to an initial $\mathrm{OD}_{600}$ of 0.75 and incubated at $37^{\circ} \mathrm{C}$ for $24 \mathrm{~h}$. A culture inoculated into PCE without MG as well as uninoculated PCE containing $6 \mathrm{~m} M$ were used as controls. Supernatants were separated from cell pellets by centrifugation at 11,400 $\times g$ at $4^{\circ} \mathrm{C}$ for $10 \mathrm{~min}$ and filter sterilized. Added to each sample was $10 \%$ (vol/vol) of a $10 \mathrm{~m} M$ DSS solution $(630 \mu \mathrm{L}$ of sample and $70 \mu \mathrm{L}$ of DSS). The samples were loaded into 5-mm tubes and analyzed on a Bruker Avance III 600 (600 MHZ for $\left.{ }^{1} \mathrm{H}\right)$. The water signal was suppressed by the excitation sculpting technique. The spectra were acquired at $298 \mathrm{~K}$, with 256 scans. The $4.37 \mathrm{ppm}$ singlet represents acetol (the singlet at 2.14 was overlapped) and the $1.14 \mathrm{ppm}$ doublet represents 1,2-propanediol (the multiplets at 3.44, 3.54, and 3.88 $\mathrm{ppm}$ were overlapped). The concentration of analytes were calculate using the DSS peak in 0 ppm as reference.

\section{Determination of MG Tolerance by Growth Kinetics}

The MG tolerance among Lactobacillus strains was characterized by using linear regression to determine (1) the bacteriostatic concentration of MG required to reduce maximum growth rate $\left(\mu_{\max }\right)$ by $50 \%$ and $(2)$ the increase in lag-time $(\lambda)$. Early stationary phase cells were standardized spectrophotometrically to an $\mathrm{OD}_{600}$ of 1.0 before being inoculated $(10 \% \mathrm{vol} / \mathrm{vol})$ into MRS containing $0,1,2$, and $3 \mathrm{~m} M$ MG. Concentrations up to $6 \mathrm{mM}$ were tested for L. brevis 367. Growth kinetics were measured spectrophotometrically at 600 $\mathrm{nm}$ every hour for $48 \mathrm{~h}$ in a flat-bottom 96-well assay plate (Corning Inc., Corning, NY) using a plate reader (Varioscan Flash, Thermo Scientific, Waltham, MA). Growth conditions included incubation of cultures at $37^{\circ} \mathrm{C}$ and shaking (180 rpm) for $10 \mathrm{~s}$ before each spectrophotometric read. The $\mu_{\max }$ and $\lambda$ were modeled using a modified Gompertz equation according to Begot et al. (1996) using a statistics package JMP (version 11.0.0, SAS Institute Inc., Cary, NC). Each strain was measured in biological triplicate, with 3 technical assay replicates each. Statistical analyses and strain-to-strain comparisons were performed using a one-way ANOVA for the concentration of MG to decrease $\mu_{\max }$ by $50 \%$ and a 2-way ANOVA for the increase in lag-time caused by 1 to $3 \mathrm{mM}$ MG. Both analyses were subject to Tukey's multiple comparison test with a $P$-value of $<0.05$ using a statistics package (GraphPad Prism v6.07, GraphPad Software, San Diego, CA).

\section{RESULTS}

\section{MG-Mediated Browning and Volatile Formation of PCE}

We observed a visual discoloration (Figure 1) and measured qualitative increase of certain volatile compounds that correlated with the addition of $\mathrm{MG}$ to PCE (Table 2). We detected volatile compounds in the headspace of Parmesan cheese extract treated with MG using SPME and GC-MS. Several short-chain aldehydes, pyrazine derivatives, and di- and tri-sulfide compounds formed as a result of MG addition.

\section{Effect of Lactobacillus Strains on a-Dicarbonyl Levels in PCE}

The ability to influence $\alpha$-dicarbonyl concentrations in PCE varied among Lactobacillus strains after 1 wk of growth, whereas 5 of the 14 strains unable to grow were omitted from this study. The concentrations of MG, glyoxal, and diacetyl were quantified in supernatants of PCE supplemented with MG after single-strain growth using o-phenyldiamine derivatization and detection by GC-MS (Figure 2). Particular interest was taken in identifying strains able to decrease MG in PCE compared with an un-inoculated control $(167 \mu M)$. Most distinctly, the strains L. brevis 367 and Lactobacillus fermentum 14931 exhibited the greatest ability to significantly reduce MG to 24.6 and $61.7 \mu M$, representing an 85 and $63 \%$ decrease, respectively. Other strains reduced MG only by 16 to $29 \%$. In comparison, the strain L. sakei 15521 was the only producer of MG, exhibiting a $60 \%$ increase. Glyoxal concentrations among strains remained equivalent and similar to the uninoculated control $(20 \mu M)$. Diacetyl concentrations were found to significantly increase compared with the uninoculated control $(42 \mu M)$ after growth of Lactobacillus casei 334, L. casei $12 \mathrm{~A}$, Lactobacillus rhamnosus GG, and L. fermentum 8289 by $46,69,76$, and $78 \%$, respectively.

\section{Lactobacillus Exhibit Thiol-Independent Metabolism of MG in Parmesan Cheese}

To determine the metabolic pathway of MG in Lactobacillus, ${ }^{1} \mathrm{H}$-nuclear magnetic resonance (NMR) was used to detect the accumulation of downstream metabolites in PCE supplemented with MG after singlestrain, high-cell-density inoculation (Figure 3). The majority of Lactobacillus strains metabolized MG to either acetol or 1,2-propanediol, representing partially and fully reduced forms of MG, respectively (Figure 4). Neither uninoculated PCE containing $6 \mathrm{mM}$ MG nor 


\section{PCE Appearance}

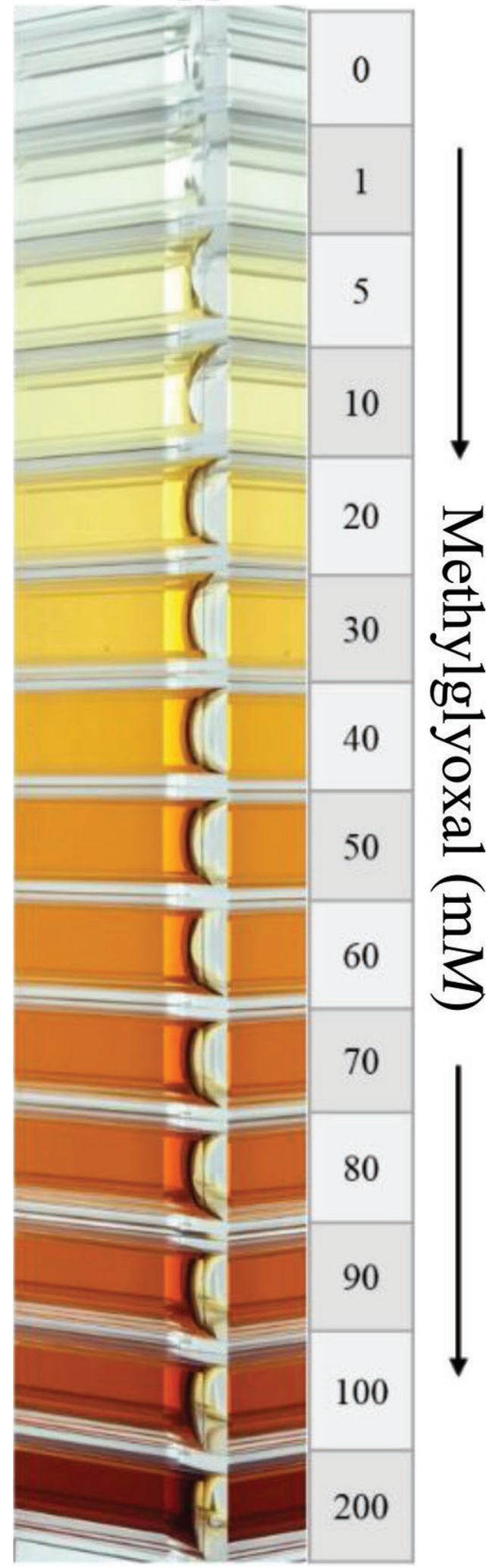

Figure 1. Appearance of Parmesan cheese extract (PCE) supplemented with increasing concentrations of methylglyoxal. strains inoculated into PCE without MG formed acetol or 1,2 propanediol. Furthermore, neither the other partially reduced form of MG, lactaldehyde, nor the glyoxalase-pathway intermediate, s-lactoylglutathione, were detected. Lactobacillus brevis 367 and L. fermentum 14931 were the only strains to reduce MG to 1,2-propanediol, whereas the majority of other strains were found to accumulate acetol. Lactobacillus brevis 367 formed exclusively 1,2-propanediol, whereas L. fermentum 14931 formed both acetol and 1,2-propanediol. The concentration of acetol accumulation varied among strains, ranging from none detected up to $4 \mathrm{~m} M$. The amounts of 1,2-propanediol formed by L. brevis 367 and L. fermentum 14931 were proportional to the amount of MG supplemented. The exception to this was Lactobacillus reuteri SD2112 and L. reuteri PTA-6475, which did not accumulate either metabolite.

\section{MG Tolerance Among Lactobacillus: Relatively High Tolerance of $L$. brevis 367}

Methylglyoxal is a biologically disruptive secondary metabolite, whose influence on the appearance and flavor of Parmesan cheese is a partial function of the cheese's ripening microbiota. We measured tolerance to MG among strains to determine whether this correlates to the differences in ability to form acetol and 1,2-propanediol. We screened Lactobacillus strains for their inherent ability to tolerate bacteriostatic concentrations of MG in rich media. Two criteria were used to assess tolerance to MG among Lactobacillus strains: (1) the concentration required to decrease maximum growth rate $\left(\mu_{\max }\right)$ by $50 \%$ and $(2)$ the increase in lag-time $(\lambda)$ due to MG (Figure 5a and 5b). Based on both these criteria, L. brevis 367 was more highly tolerant relative to other strains, requiring the greatest concentration of MG $(5.2 \mathrm{mM})$ to decrease $\mu_{\max }$ by $50 \%$ while also exhibiting the smallest increase in lag time (additional $5 \mathrm{~h}$ due to $3 \mathrm{mM}$ ). All other strains required only 1.0 to $2.1 \mathrm{~m} M \mathrm{MG}$ to decrease $\mu_{\max }$ by $50 \%$ and incurred lagtime increases of 9 to $24 \mathrm{~h}$ in the presence of $3 \mathrm{mM} \mathrm{MG}$.

\section{DISCUSSION}

Microbial production of MG has been implicated for undesirable browning of Parmesan cheese during ripening and cold storage processes, causing economic detriment to the industry. Consistent color is critical for the successful manufacturing of Parmesan and the use of culture adjuncts to mitigate MG-based browning presents a natural solution, unlike the use of chemical reducing agents and bleaching applications. We demonstrated the effects of MG on discoloration and volatile formation in PCE. Moreover, we determined the influ- 
Table 2. Volatile compounds detected by solid-phase microextraction GC-MS found to increase in concentration in accordance to increasing exogenous addition of methylglyoxal (1, 10, 50, and $100 \mathrm{mM}$ ) to Parmesan cheese extract $(\mathrm{PCE})$

\begin{tabular}{ll}
\hline Compound & Organoleptic property $^{1}$ \\
\hline Acetaldehyde & Wine-like \\
2-Methylpropanal (isobutyraldehyde) & Cheese \\
2,3 Butanedione (diacetyl) & Butter \\
2-Butananone & Butterscotch, ethereal \\
3-Methylbutanal (isovaleraldehyde) & Fruity, peach, amylic \\
2-Methylbutanal (2-methylbutyraldehyde) & Chocolate, coffee \\
Dimethyl disulfide & Garlic, onion \\
3-Methyl-2-butenal & Cherry \\
Butyric acid & Rancid \\
4-Methyl pyrazine & Roasted, nutty \\
3-Methylbutyric acid (isovaleric acid) & Fatty \\
2-Methyl-hexanoic acid (2-methylcaproic acid) & Lard \\
3-Methylthiopropanal (methional) & Earthy, potato, tomato \\
Dimethylpyrazine & Roasted, nutty \\
5-Methyl-2(5H)-furanone & Oily, nutty \\
5-Methyl-2-furancarboxaldehyde (2-furfural) & Almond, woody, sweet \\
Benzaldehyde & Almond, cherry, sweet \\
Dimethyl trisulfide & Meaty, sulfurous \\
2-Acetyl-5-methylfuran & Nutty \\
Benzeneacetaldehyde (phenylacetaldehyde) & Apple, apricot, melon \\
2-Hydroxy-benzaldehyde (salicylaldehyde) & Almond, spicy \\
2-Ethyl-dimethyl-pyrazine & Almond, caramel, tobacco \\
Benzothiazole & Sulfurous \\
\hline
\end{tabular}

${ }^{1}$ As described by Sigma-Aldrich Flavors and Fragrance Ingredients Catalog (Sigma-Aldrich, St. Louis, MO).

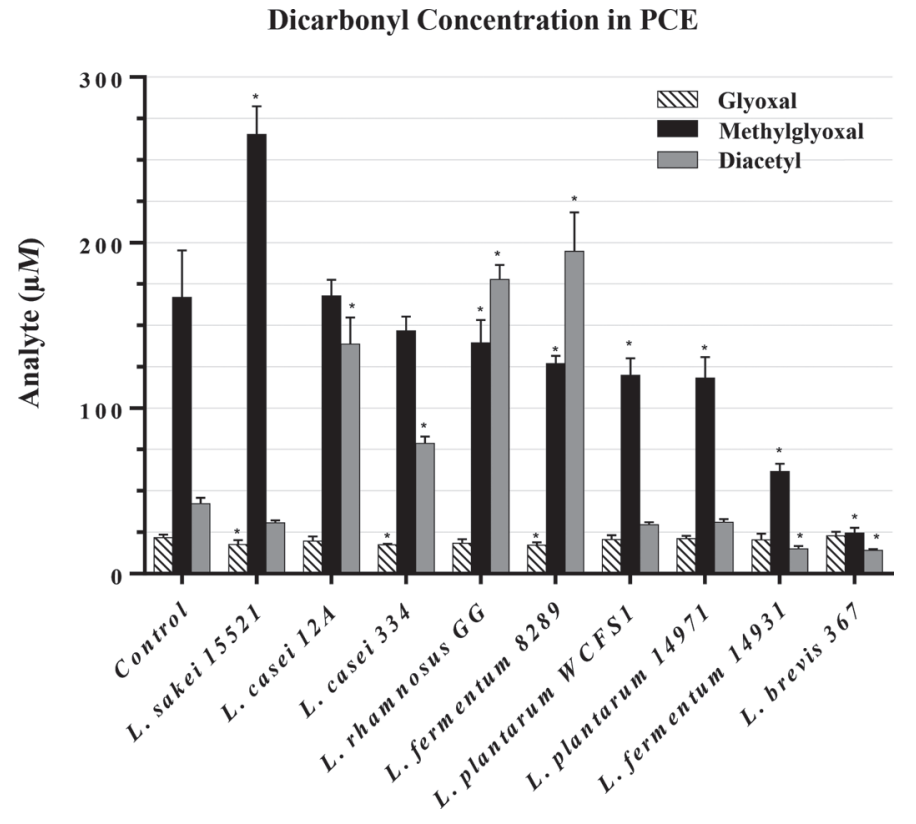

Figure 2. Mean concentration(s) of $\alpha$-dicarbonyls glyoxal, methylglyoxal, and diacetyl found in supernatants of Parmesan cheese extract (PCE) supplemented with $1 \mathrm{~m} M$ methylglyoxal after 1 wk growth at $37^{\circ} \mathrm{C}$. The control was uninoculated PCE supplemented with $1 \mathrm{mM}$ methylglyoxal. Statistically different levels from the control $(*)$ were determined using a paired $t$-test with an $\alpha$ of $<0.05$. Error bars represent SD of the mean. L. = Lactobacillus. ence of different Lactobacillus strains on modulating $\alpha$-dicarbonyl concentrations in PCE and their propensity for a thiol-independent detoxification of MG. This study used PCE to simulate constituents in Parmesan that would readily react with MG while being able to use it as a sterile growth media for single-strain evaluation. Although ripening and cold storage of Parmesan takes place at cooler temperatures, strains were evaluated at their optimal growth temperature of $37^{\circ} \mathrm{C}$ to accelerate fermentation.

In addition to MG-based browning of PCE, we observed the formation of several volatile compounds. The short-chain aldehydes (2-methylpropanal, 2-methylbutanal, 3-methylbutanal, 3-methyl-2-butenal, 3-methylthiopropanal) may result from nucleophile substitution of either carbonyl group of MG by amino groups of AA as well as alkane or alkene hydrocarbons. The pyrazine (dimethylpyrazine and 2-ethyl-dimethyl-pyrazine) formation can occur from nucleophilic substitution of MG by primary amines to form aminoacetone, whereby condensation can form nitrogenous cyclic compounds with various side chains. A study published by PripisNicolau et al. (2000) described similar products to the ones found here from reactions between $\alpha$-dicarbonyls and AA present in wine. Volatile compounds, including 2,5-dimethylpyrazine, 2,6-dimethylpyrazine, methional, dimethyl disulfide, 2-methylpropanal, 2-methylbutanal, 3-methylbutanal, and benzaldehyde, formed when $\alpha$-dicarbonyls reacted with cysteine, methionine, 
valine, leucine, isoleucine, and phenylalanine, respectively. These compounds are known to impart nutty, toasted, hazelnut, earthy, sulfurous, fruity, and sweet notes, which are interesting for their complex organoleptic properties; however, their contribution toward Parmesan flavor remains to be evaluated.

The contribution of MG to flavor development of fermented foods and beverages has previously garnered interest. A study by Griffith and Hammond (1989) demonstrated the formation of Swiss cheese flavor components by reacting AA with glyoxal and MG. In relation to this goal, Bednarski and Hammond (1990) developed a method for screening Lactobacillus for their dicarbonyl production capacity with the objective of isolating strains that would accelerate carbonyl-amine reactions for developing cheese flavor. Methylglyoxal (pyruvaldehyde) is listed in the Code of Federal Regulations (21CFR172) as a synthetic flavoring substance or adjuvant permitted for direct addition to food for human consumption (US Food and Drug Administration, 2016) and denoted as having generally recognized as safe (GRAS) status by the Flavor and Extract Manufacturers Association (FEMA No. 2969; FEMA, 1965).
Despite causing undesirable browning of Parmesan cheese, the utility of reactive $\alpha$-dicarbonyl compounds to intensify the color and flavor of cheese remains a potential value added technology to be explored.

The major source of MG in biological systems is from the degradation of the glycolytic intermediate, DHAP, which is thought to occur primarily when sugar levels are in excess or when inorganic phosphate levels are limiting (Cooper, 1984). The only known enzyme that specifically catalyzes MG formation from DHAP is MG synthase (Sousa Silva et al., 2013). It is likely that MG formation in Parmesan is linked to the discrepancy between ripening microbiota associated with 2 different manufacturing styles: direct and brine salting (McDonald, 1992). We find the strain L. sakei 15521 exhibits an ability to produce MG while growing in PCE. According to the GenBank database, 17 Lactobacillus species are annotated to contain the $\sim 140$-AA protein MG synthase (encoded by the gene $m g s A$ ), which catalyzes the conversion of DHAP to MG: L. sakei, L. curvatus, L. coryniformis, L. pobuzihii, L. shenzhenesis, L. composti, L. graminis, L. bifermentans, L. rennini, $L$. perolens, L. saniviri, L. nasuensis, L. acidipscis, L. vini,
1. L. brevis 367

2. L. fermentum 14931

3. L. fermentum 8289

4. L. sakei 15521

5. L. sakei $23 \mathrm{~K}$

6. L. rhamnosus GG

7. L. reuteri $\mathrm{SD} 2112$

8. L. reuteri PTA-6475

9. L. plantarum WCFS1

10. L. plantarum 14971

11. L. curvatus 112309-12

12. L. helveticus CNRZ32

13. L. casei 334

14. L. casei $12 \mathrm{~A}$

15. Uninoculated Control
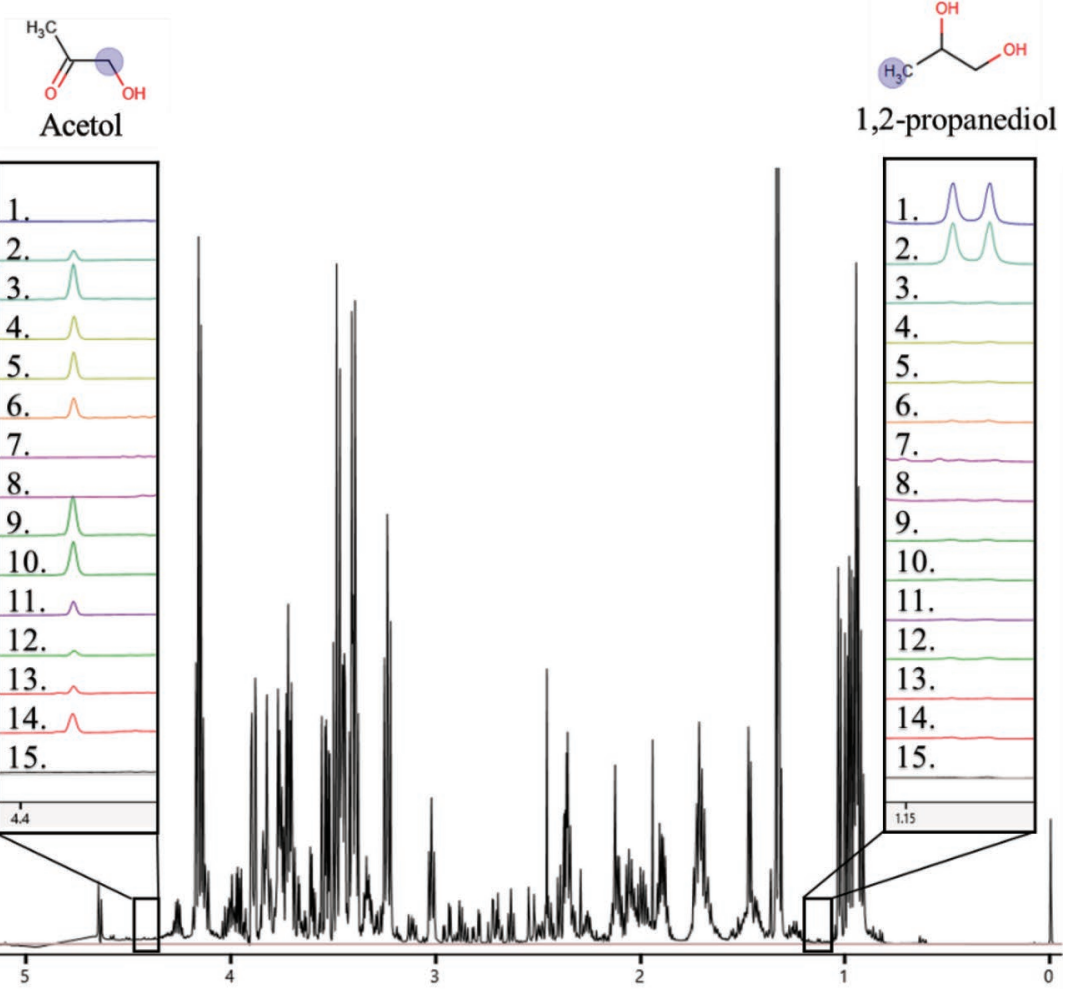

Figure 3. ${ }^{1} \mathrm{H}-\mathrm{Nuclear}$ magnetic resonance spectra of supernatants from Lactobacillus grown in Parmesan cheese extract supplemented with methylglyoxal. The $4.37 \mathrm{ppm}$ singlet represents acetol (the singlet at 2.14 was overlapped) and the doublet at 1.14 ppm represents 1,2 -propanediol (the multiplets at 3.44,3.54, and $3.88 \mathrm{ppm}$ were overlapped). The horizontal scale represents the chemical shift (ppm). Color version available online. 


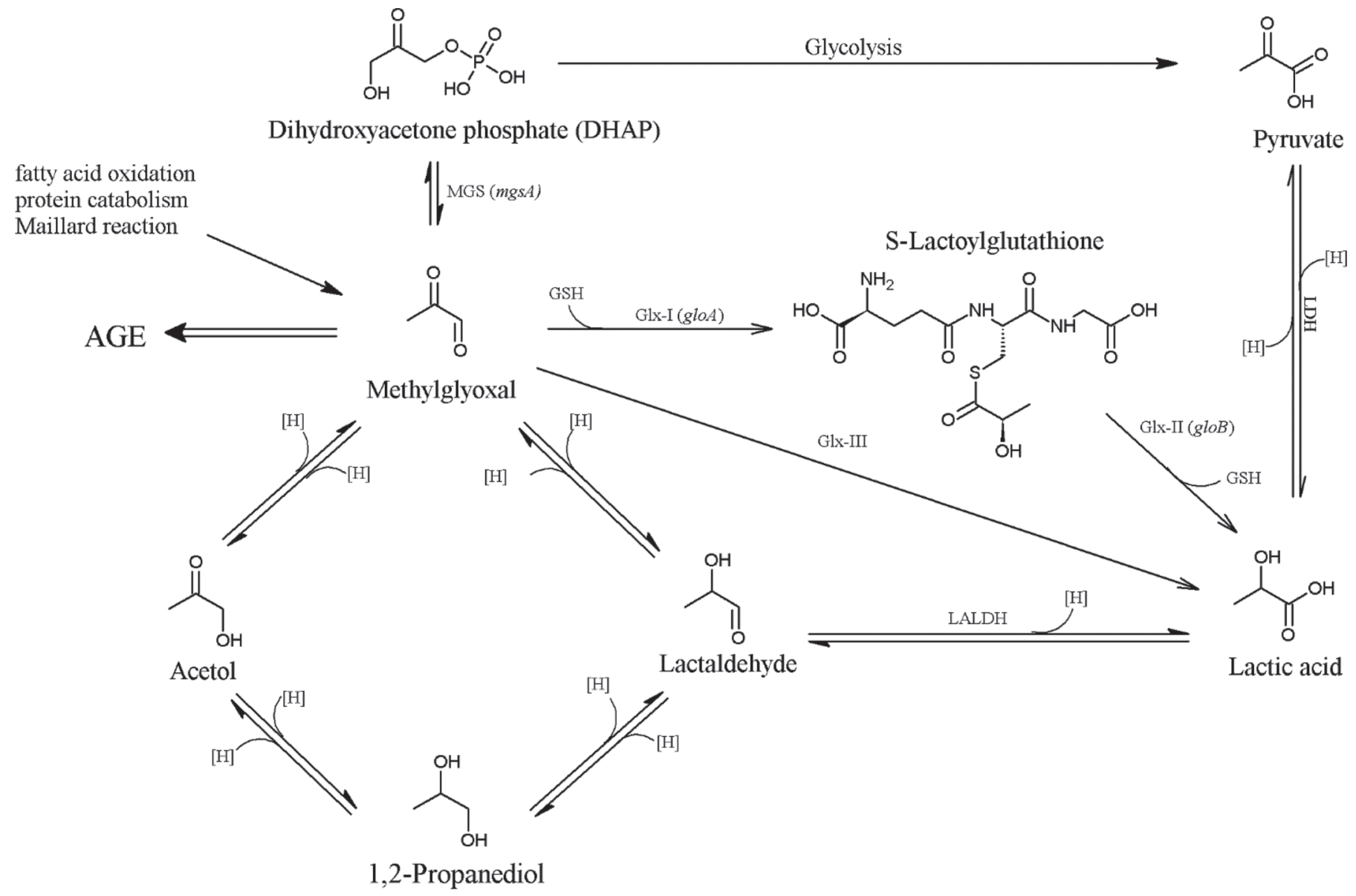

Figure 4. Precursors and end products of methylglyoxal metabolism. MGS = methylglyoxal synthase; Glx-I = glyoxalase I (lactoylglutathione lyase); Glx-II = glyoxalase II (hydroxyacylglutathione hydrolase); Glx-III = glyoxalase III; LALDH = lactaldehyde dehydrogenase; LDH = lactate dehydrogenase; GSH = reduced glutathione; AGE = advanced glycation end products.

L. harbinensis, L. fuchuensis, and L. rogosae (Benson et al., 2017). Further studies are required to determine if $m g s A$-containing species are implicated in MGmediated browning of Parmesan cheese. However, the use of $m g s A$ as a genetic bio-marker for the detection and control of offending microflora could be useful for improving the quality control of affected cheeses.

We determined strain-specific tolerance to bacteriostatic concentrations of MG to implicate metabolic activity on MG. Four major routes of MG toxicity are commonly accepted: (1) direct inhibitory effect on enzymes, (2) direct genotoxicity causing cell death, (3) indirect toxicity by the depletion of glutathione or other thiols, and (4) oxidative stress from the production of free radicals and reactive oxygen species (Kalapos, 2008). MG has been well documented to cause posttranslational modification of proteins at lysine, arginine, and cysteine residues and is a known mutagen, causing DNA mismatch and DNA-protein cross-linking
(Lo et al., 1994; Frischmann et al., 2005). These reactions are interesting for their formation of MG-derived AGE because they have been implicated, by and large, for physiological detriment as well as pathological changes in aging, diabetes, and other disorders (Finkel and Holbrook, 2000; Ramasamy et al., 2006; Thornalley, 2008).

A well-studied detoxification adduct is the thioldependent glyoxalase pathway, which serves to protect against MG accumulation and as an energy-futile glycolytic bypass (Booth et al., 2003; Suttisansanee and Honek, 2011). The pathway requires the thiol group of glutathione, and consists of a 2-step reaction catalyzed by glyoxalase $\mathrm{A}$ and glyoxalase $\mathrm{B}$, forming the intermediate S-lactoylglutathione and the end product lactic acid, respectively. It is generally considered that glutathione biosynthesis is absent from gram-positive Firmicutes, such as Lactobacillus (Fernandes and Steele, 1993; Steele and Wiederholt, 1994); however, low mo- 
A. Methylglyoxal concentration to decrease $\mu \max$ by $50 \%$

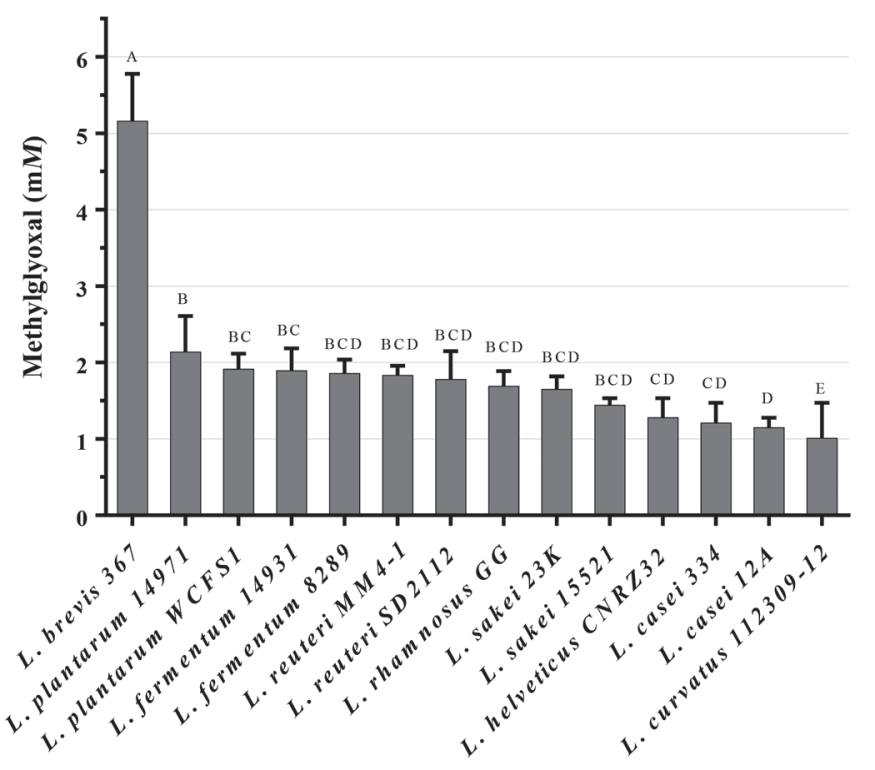

B. Increase in lag-time due to methylglyoxal exposure
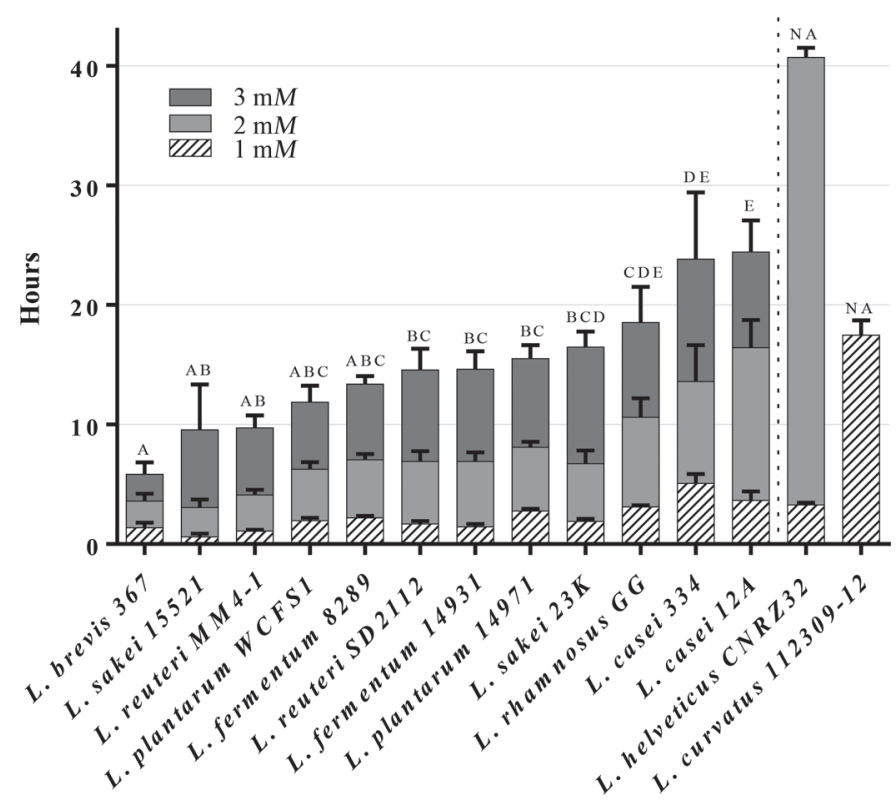

Figure 5. Inherent tolerance to methylglyoxal among Lactobacillus. (A) The mean concentration of methylglyoxal required to decrease maximum growth rate $\left(\mu_{\max }\right)$ by $50 \%$. (B) The mean increase in lag time due to 1 to $3 \mathrm{mM}$ methylglyoxal. Strains containing the same letter are considered not statistically different as per Tukey's honestly significant difference with $\alpha<0.05$. Error bars represent SD of the mean.

lecular weight thiols such as cysteine or methanethiol may act as viable substitutes in forming thiol-MG conjugates as similarly demonstrated by bacillithiol utilization in Bacillus subtilis (Gaballa et al., 2010). In this study, we did not detect the formation of the MG metabolic intermediate S-lactoylglutathione among any strains, which is consistent with the literature.

Cytosolic acidification protects bacteria against strong electrophiles such as MG and is regarded as the predominant defense against toxicity; however, the mechanisms by which it does so remain unclear. It is hypothesized to minimize electrophile-macromolecule interaction and galvanize various repair systems (Ferguson et al., 1998). To this end, Lactobacillus inherently allow their intracellular $\mathrm{pH}$ to decline as low as 4.4 while producing lactic acid as the major metabolic end product. Although S-lactoylglutathione is a detoxification intermediate in the thiol-dependent glyoxalase system, it is also an activator of the KefB/KefC potassium efflux system in Escherichia coli. The loss of potassium by the KefB/KefC system is accompanied by an influx of sodium and protons, the latter subsequently lowering intracellular $\mathrm{pH}$ and offering protection against electrophiles (Ferguson et al., 1998). Mutants lacking KefB/ KefC systems are highly sensitive to MG compared with the parent strain (Ferguson et al., 1993). Consistent with previous work in E. coli, a similar system was found to occur with the KhtSTU potassium efflux pump in B. subtilis, albeit activation was attributed to S-lactoylbacillithiol instead of S-lactoylglutathione (Chandrangsu et al., 2014). Although we found the strains included in this study do not produce S-lactoylglutathione as a metabolic intermediate, the inherently low intracellular $\mathrm{pH}$ of Lactobacillus likely offers protection from MG toxicity.

We found that Lactobacillus can metabolize MG by a thiol-independent manner by reducing it to acetol and 1,2-propanediol. Thiol-independent MG detoxification is catalyzed by broad-specificity aldo-keto reductases, several of which have been characterized in E. coli (Ko et al., 2005). Previously determined in B. subtilis, the aldo-keto reductase, YhdN, that converts MG to acetol was reported as the single most important detoxification route for this organism, by comparing MG sensitivity between $y h d N$ null, glyoxalase null, and bacillithiol null strains (Chandrangsu et al., 2014).

We observed L. brevis 367 and L. fermentum 14931 exhibit the ability to significantly decrease MG concentrations after $1 \mathrm{wk}$ of growth in PCE containing 1 $\mathrm{m} M \mathrm{MG}$. Both strains also decrease diacetyl concentrations, which suggests broader redox ability toward $\alpha$-dicarbonyls. We determined the metabolic end products of MG, acetol, and 1,2 propanediol, after high-cell density inoculation of PCE containing $6 \mathrm{mM} \mathrm{MG}$ by ${ }^{1} \mathrm{H}-$ NMR. Lactobacillus brevis 367 is the only strain herein to reduce MG entirely to 1,2-propanediol rather than forming the partially reduced intermediate, acetol. This finding correlates with the relatively high MG tolerance of L. brevis 367 compared with other strains in this 
study. Lactobacillus fermentum 14931 also converts MG to 1,2-propanediol; however, we observed that acetol partially accumulates and the strain's tolerance to MG is similar to others tested. Therefore, it is of interest to identify enzymes unique to L. brevis 367 that catalyze this reaction and to determine to what extent they contribute toward MG tolerance. An in silico approach to identify LAB containing genetic elements relating to MG metabolism in L. brevis 367 could hasten screening processes and lead to new insights toward developing a microbial means of controlling MG-mediated reactions in cheese.

The exceptions to this finding are L. reuteri SD2112 and L. reuteri PTA-6475, in which acetol, 1,2-propanediol, lactaldehyde, and S-lactoylglutathione were not detected. The absence of metabolites detected in $L$. reuteri could indicate the formation of alternative thiol-MG intermediates in the glyoxalase pathway or the direct conversion of MG to lactic acid. Low-molecular-weight thiols such as cysteine or methanethiol may act as viable substitutes for glutathione in forming thiol-MG conjugates to participate in a glyoxalase-dependent conversion to lactic acid, similar to bacillithiol utilization in B. subtilis (Gaballa et al., 2010). Alternatively, the direct conversion of MG to lactic acid can be catalyzed by a thiol-independent glyoxalase III enzyme previously only characterized in E. coli (Misra et al., 1995; Subedi et al., 2011). In B. subtilis 3 structural homologs (YdeA, YfkM, and YraA) to the glyoxalase III found in E. coli contribute to MG resistance in a thiol-independent manner (Chandrangsu et al., 2014).

\section{CONCLUSIONS}

A major metabolic pathway of MG in Lactobacillus carries out thiol-independent reduction of MG to acetol and 1,2-propanediol. The ability of Lactobacillus to modulate the $\alpha$-dicarbonyls MG and diacetyl, and to a lesser extent, glyoxal, in PCE is a strain-specific trait. The distinct reduction of MG to 1,2-propanediol exhibited by L. brevis 367 likely contributes to the organism's high tolerance to bacteriostatic concentrations of MG. Future interests in determining the proteins most active on MG might be fulfilled by the in silico identification of LAB reductases comparable to those found in L. brevis 367 . Hence, LAB exhibiting similar redox capabilities on MG are attractive natural solutions to MG-mediated browning observed in Parmesan and would warrant further evaluation in a more authentic cheese model system. The role of Lactobacillus in MGmediated browning of Parmesan cheese will continue to have broader implications toward the influence of $\alpha$-oxoaldehydes on the quality and consistency of LABbased fermented food and beverages.

\section{ACKNOWLEDGMENTS}

We thank the Wisconsin Alumni Research Foundation (Madison, Wisconsin) and the Fundação de Amparo à Pesquisa do Estado de São Paulo, FAPESP (São Paulo, Brazil) grant \#2012/12847-5 and \#2015/025348 for their support. This study made use of the National Magnetic Resonance Facility at Madison, Wisconsin, which is supported by NIH (Bethesda, MD) grants P41 GM103399 (NIGMS) and P41GM66326 (NIGMS). Additional equipment was purchased with funds from the University of Wisconsin (Madison), the NIH (RR02781, RR08438), the NSF (Alexandria, Virginia), DMB8415048, OIA-9977486, BIR-9214394, and the USDA (Washington, DC).

\section{REFERENCES}

Bednarski, W., and E. G. Hammond. 1990. Selection of Lactobacillus mutants for their $\alpha$-dicarbonyl production. J. Dairy Sci. 73:1450 1453.

Begot, C., I. Desnier, J. Daudin, J. Labadie, and A. Lebert. 1996. Recommendations for calculating growth parameters by optical density measurements. J. Microbiol. Methods 25:225-232.

Benson, D. A., M. Cavanaugh, K. Clark, I. Karsch-Mizrachi, D. J. Lipman, J. Ostell, and E. W. Sayers. 2017. GenBank. Nucleic Acids Res. 45:D37-D42.

Booth. I. R., G. P. Ferguson, S. Miller, C. Li, B. Gunasekera, and S. Kinghorn. 2003. Bacterial production of methylglyoxal: A survival strategy or death by misadventure? Biochem. Soc. Trans. 31:1406-1408.

Broadbent, J. R., M. F. Budinich, and J. L. Steele. 2011. Cheese: NSLAB. Pages 639-644 in Encyclopedia of Dairy Science. 2nd ed. J. Fuquay, P. Fox, and P. McSweeney, ed. Academic Press, Burlington, MA.

Chandrangsu, P., R. Dusi, C. J. Hamilton, and J. D. Helmann. 2014. Methylglyoxal resistance in Bacillus subtilis: Contributions of bacillithiol-dependent and independent pathways. Mol. Microbiol. 91:706-715.

Chaplen, F. W., W. E. Fahl, and D. C. Cameron. 1996. Method for determination of free intracellular and extracellular methylglyoxal in animal cells grown in culture. Anal. Biochem. 238:171-178.

Compendium of International Analysis of Methods. 2010. Methods for the Determination of Alpha-Dicarbonyl Compounds of Wine by GC After Derivitization by 1,2-Diaminobenzene. International Organisation of Vine and Wine. OIV-MA-AS315-21.

Cooper, R. A. 1984. Metabolism of methylglyoxal in microorganisms. Annu. Rev. Microbiol. 38:49-68.

Divine, R. D., and S. A. Rankin. 2013. Short communication: Reducing agents attenuate methylglyoxal-based browning in Parmesan cheese. J. Dairy Sci. 96:6242-6247.

Divine, R. D., D. Sommer, A. Lopez-Hernandez, and S. A. Rankin. 2012. Short communication: Evidence for methylglyoxal-mediated browning of Parmesan cheese during low temperature storage. J. Dairy Sci. 95:2347-2354.

FEMA. 1965. GRAS substances: Recent progress in the consideration of flavoring ingredients under the food additives amendment. J. Institute Food Technologists 19:151-197.

Ferguson, G. P., A. W. Munro, R. M. Douglas, D. McLaggan, and I. R. Booth. 1993. Activation of potassium channels during metabolite detoxification in Escherichia coli. Mol. Microbiol. 9:1297-1303.

Ferguson, G. P., S. Tötemeyer, M. J. MacLean, and I. R. Booth. 1998 Methylglyoxal production in bacteria: Suicide or survival? Arch. Microbiol. 170:209-218.

Fernandes, L., and J. L. Steele. 1993. Glutathione content of lactic acid bacteria. J. Dairy Sci. 76:1233-1242. 
Finkel, T., and N. J. Holbrook. 2000. Oxidants, oxidative stress and the biology of ageing. Nature 408:239-247.

Frischmann, M., C. Bidmon, J. Angerer, and M. Pischetsrieder. 2005. Identification of DNA adducts of methylglyoxal. Chem. Res. Toxicol. 18:1586-1592

Gaballa, A., G. L. Newton, H. Antelmann, D. Parsonage, H. Upton, M. Rawat, A. Claiborne, R. C. Fahey, and J. D. Helmann. 2010. Biosynthesis and functions of bacillithiol, a major low-molecularweight thiol in Bacilli. Proc. Natl. Acad. Sci. USA 107:6482-6486.

Griffith, R., and G. Hammond. 1989. Generation of Swiss cheese flavor components by the reaction of amino acids with carbonyl compounds. J. Dairy Sci. 72:604-613.

Kalapos, M. P. 2008. The tandem of free radicals and methylglyoxal. Chem. Biol. Interact. 171:251-271.

Ko, J., I. Kim, S. Yoo, B. Min, K. Kim, and C. Park. 2005. Conversion of methylglyoxal to acetol by Escherichia coli aldo-keto reductases. J. Bacteriol. 187:5782-5789.

Lo, T. W., M. E. Westwood, A. C. McLellan, T. Selwood, and P. J. Thornalley. 1994. Binding and modification of proteins by methylglyoxal under physiological conditions. A kinetic and mechanistic study with $\mathrm{N}$ alpha-acetylarginine, $\mathrm{N}$ alpha-acetylcysteine, and $\mathrm{N}$ alpha-acetyllysine, and bovine serum albumin. J. Biol. Chem. 269:32299-32305

McDonald, S. 1992. Role of a-dicarbonyl compounds produced by lactic acid bacteria on the flavor and color of cheese. PhD Thesis. Department of Food Science, University of Wisconsin-Madison.

Misra, K., A. B. Banerjee, S. Ray, and M. Ray. 1995. Glyoxalase III from Escherichia coli: A single novel enzyme for the conversion of methylglyoxal into D-lactate without reduced glutathione. Biochem. J. 305:999-1003.

Nemet, I., L. Varga-Defterdarović, and Z. Turk. 2006. Methylglyoxal in food and living organisms. Mol. Nutr. Food Res. 50:1105-1117.

Pripis-Nicolau, L., G. de Revel, A. Bertrand, and A. Maujean. 2000. Formation of flavor components by the reaction of amino acid and carbonyl compounds in mild conditions. J. Agric. Food Chem. 48:3761-3766.

Ramasamy, R., S. F. Yan, and A. M. Schmidt. 2006. Methylglyoxal comes of AGE. Cell 124:258-260.

Sousa Silva, M., R. A. Gomes, A. E. Ferreira, A. Ponces Freire, and C. Cordeiro. 2013. The glyoxalase pathway: the first hundred years... and beyond. Biochem. J. 453:1-15.

Steele, J. L., and K. M. Wiederholt. 1994. Glutathione accumulation in lactococci. J. Dairy Sci. 77:1183-1188.

Subedi, K. P., D. Choi, I. Kim, B. Min, and C. Park. 2011. Hsp31 of Escherichia coli K-12 is glyoxalase III. Mol. Microbiol. 81:926-936.

Suttisansanee, U., and J. F. Honek. 2011. Bacterial glyoxalase enzymes. Semin. Cell Dev. Biol. 22:285-292.

Thornalley, P. J. 2008. Protein and nucleotide damage by glyoxal and methylglyoxal in physiological systems-Role in ageing and disease. Drug Metabol. Drug Interact. 23:125-150.

US Food and Drug Administration. 2016. Food Additives Permitted For Direct Addition To Food For Human Consumption. 21CFR part 172. Fed. Regist. Sec. 172.515.

Yann, D., H. Didier, and B. Daniel. 2005. Utilisation of the experimental design methodology to reduce browning defects in hard cheeses technology. J. Food Eng. 68:481-490. 